\title{
MyWave: Sistemas locales de predicción de oleaje por conjuntos
}

Ola tras ola siguió Ralph aquel subir y bajar hasta que algo propio del carácter distante del mar le embotó la mente.

El señor de las moscas - WILLIAM GOLDING

La información meteorológica es de vital importancia para el apoyo a la navegación marítima. La dirección e intensidad de los vientos, así como la intensidad y dirección del oleaje, son cruciales y están interrelacionados. AEMET, en colaboración con el Organismo Público Puertos del Estado desarrolla y explota sistemas operativos de predicción del oleaje basados en técnicas de modelización para la predicción en las zonas oceánicas de nuestro entorno. En el marco de la Organización Meteorológica Mundial, AEMET contribuye a la creación de capacidad en meteorología marítima en los países de África occidental y Macaronesia. Dentro de estos contextos, el proyecto europeo MyWave, liderado por el Instituto Meteorológico Noruego, fue creado con el objetivo principal de sentar las bases para un Centro de Servicios Marinos que incluyese el oleaje, identificando para ello cuatro áreas en las que era necesaria la investigación: la asimilación de datos, la mejora del modelo, el establecimiento de protocolos estándar de validación y la predicción por conjuntos. En este capítulo se describe el trabajo realizado para el desarrollo de estos sistemas de predicción de oleaje por conjuntos en puertos españoles, así como los resultados.

Palabras clave: sistemas locales de predicción de oleaje por conjuntos, proyecto MyWave, meteorología marítima. 


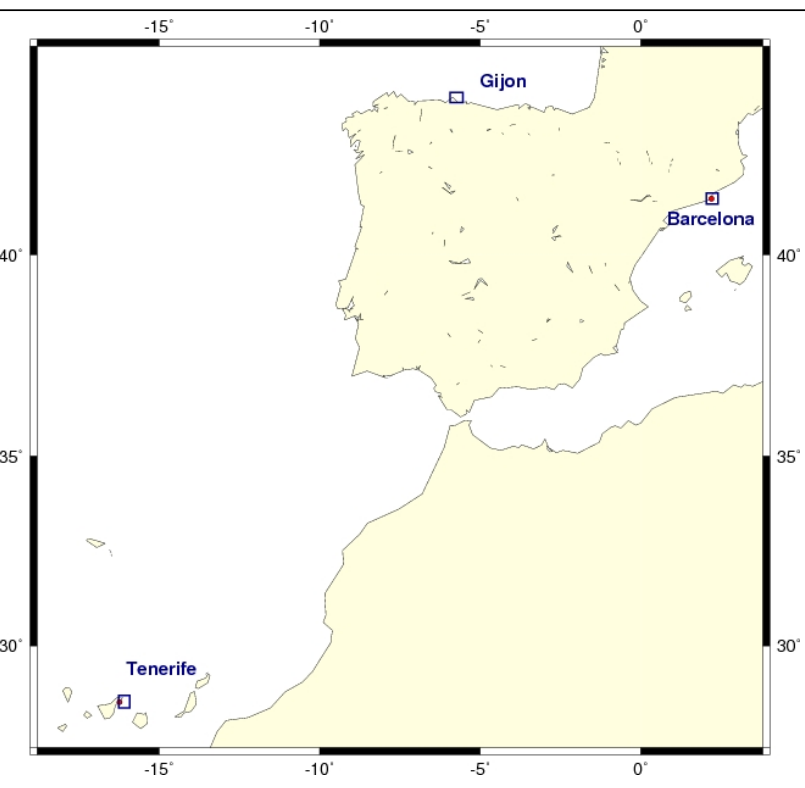

Figura 25.1: Posición de los tres sistemas de predicción de oleaje por conjuntos (WAVE-EPS), para los puertos de Gijón, Tenerife y Barcelona. En rojo, boyas de la red de Puertos del Estado dentro de las regiones de estos sistemas y utilizadas para la validación.

\subsection{SPC de olas}

El proyecto europeo FP7 MyWave [9] (2012-2014), liderado por el Instituto Meteorológico Noruego (https://www.met.no/), fue creado con el objetivo principal de sentar las bases para un Centro de Servicios Marinos que incluyese el oleaje, identificando para ello cuatro áreas en las que era necesaria la investigación: la asimilación de datos, la mejora del modelo, el establecimiento de protocolos estándar de validación y la predicción por conjuntos.

Para la mejora de la predicción por conjuntos, se desarrollaron sistemas de oleaje llamados WAVE-EPS [8] enfocados a las costas europeas en dos áreas: en el Atlántico Norte y en el mar Mediterráneo. Anidados en estos sistemas Agencia Estatal de Meteorología (AEMET) desarrolló, en colaboración con Puertos del Estado, tres sistemas de predicción de oleaje por conjuntos para los puertos de Gijón,Tenerife y Barcelona

Los tres sistemas empezaron a funcionar de forma operativa a mediados del proyecto (2013), generando los productos específicos de la predicción por conjuntos, aunque no se diseminan públicamente.

Para definir un sistema de predicción de oleaje en un área limitada se empieza por definir la malla (sec. 10.6 en la página 141) y la batimetría. Para calcular la evolución del espectro del oleaje, el modelo necesita unas condiciones iniciales y, durante la integración, campos de viento a $10 \mathrm{~m}$ y condiciones de contorno.

El modelo da como salida el espectro completo y los parámetros integrados de oleaje. Los principales parámetros son:

- Altura significativa: altura media del tercio mayor de todas las olas observadas.

- Periodo medio: periodo medio de todos los grupos de ondas que, superpuestas, forman el oleaje.

- Periodo de pico: periodo del grupo de ondas con más energía .

- Dirección media: media de las direcciones de propagación del grupo de ondas.

\subsection{Configuración de los sistemas locales WAVE-EPS}

Los sistemas para los puertos utilizaron el modelo de alta resolución Simulating Waves Nearshore, SWAN [2], desarrollado por la universidad de Delft especializado en el tratamiento del oleaje cerca de la costa.

Debido a esto, la batimetría jugó un papel importante en la configuración de los sistemas. Respecto a la configuración del WAVE-EPS local, fue determinada por modelo regional WAVE-EPS al que estaba anidado, utilizando el mismo viento y las condiciones de contorno (espectros de energía) generadas por el sistema regional.

Descripción estadística del campo de olas. La naturaleza matemática de la descripción de las olas de superficie hace necesaria una descripción estadística para sintetizar la información. Los parámetros estadísticos que representan un campo de olas caracterizan las condiciones existentes durante un cierto periodo de tiempo y para cierta extensión espacial.

El descriptor más utilizado del campo de olas es el espectro de densidad de energía en dirección y frecuencia, $E(f, \Theta)$, donde $f$ es la frecuencia y $\Theta$ la dirección de propagación. 


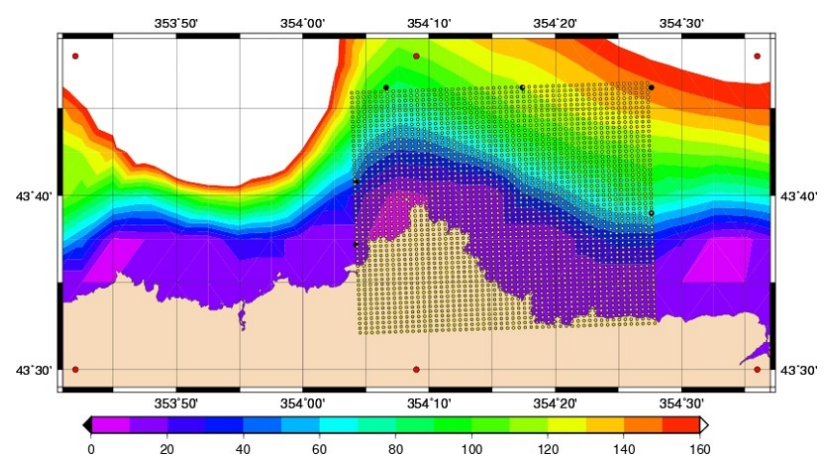

Figura 25.2: Configuración del sistema local WAVEEPS de Gijón: malla computacional (puntos amarillos), campos de viento (puntos rojos) y condiciones de contorno (puntos negros). La batimetría (sombreado en colores, de 0 a 160 metros de profundidad) muestra los límites de aguas someras y hasta dónde influiría en oleaje.

\subsubsection{Descripción de los modelos locales del Atlántico}

Los dos sistemas locales WAVE-EPS implementados en el Atlántico reciben los forzamientos del UK MetOffice (http://www. metoffice.gov.uk/): el viento del sistema MOGREPs-UK y las condiciones de contorno de su modelo regional WAVE-EPS. Este modelo usa una malla llamada Spherical Multi-Cell (SMC), que tiene una resolución variable cerca de la costa (ver página 375). Debido a ello, las condiciones de contorno no están espaciadas de forma regular.

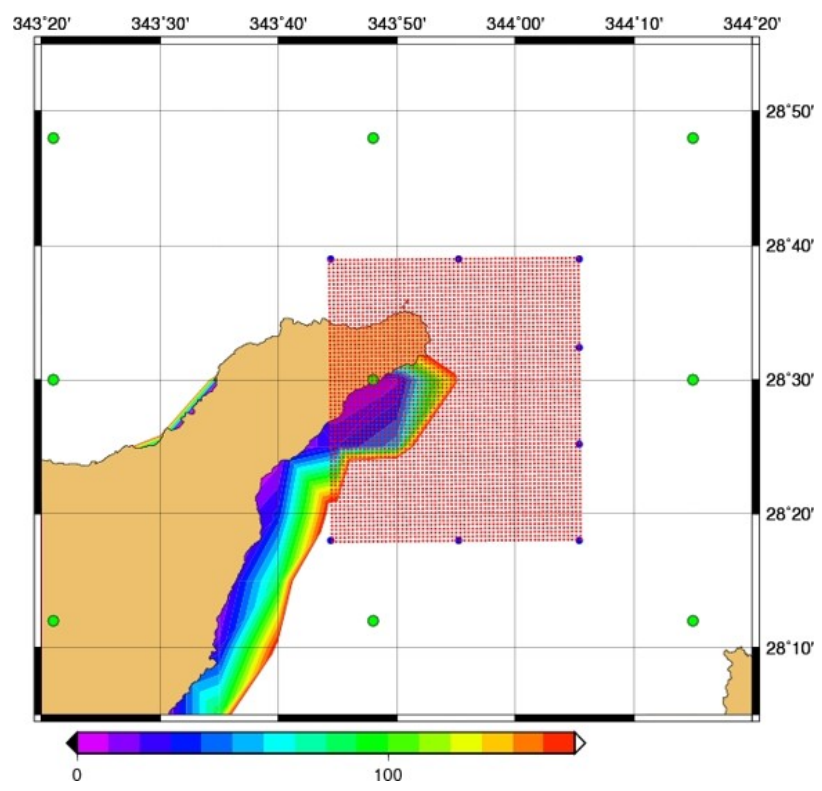

Figura 25.3: Configuración del sistema local WAVEEPS de Tenerife: malla computacional (puntos rojos), campos de viento (puntos verdes) y condiciones de contorno (puntos azules). Batimetría como en la Figura 25.2 .
El sistema de Gijón (Figura 25.2) tiene una malla computacional de 51×51 puntos, con una resolución de $629 \times 511$ metros cada celda (O -5,93, E -5,54, S 43,54, N 43,77). Los campos de viento tienen una resolución espacial de $0,45^{\circ} \times 0,30^{\circ}$.

El sistema de Tenerife (Figura 25.3) tiene una malla computacional de $61 \times 71$ puntos con celdas de resolución de 571x554 metros (O -16,26, E -15,90, S 28,30, N 28,65). Los campos de viento tendrán una resolución espacial de $0.45^{\circ} \mathrm{x} 0.30^{\circ}$.

\subsubsection{Descripción del wave-EPS del Atlántico}

El sistema regional del Atlántico generado por el MetOffice, el UKMO wave-EPS [4] está forzado por los dos EPS atmosféricos que utiliza el MetOffice: el sistema global (MOGREPS-G) y el de área limitada MOGREPS-UK [3] (ver capítulo 23 en la página 361). El modelo de oleaje utilizado será el WAVEWATCH III (WW3 [10]). Debido a que utilizan los forzamientos del UKMO-wave EPS, los sistemas locales de Gijón y Tenerife funcionan con la misma resolución temporal que el sistema del MetOffice, definido con:

- Cuatro ciclos diarios: 00/06/12/18 UTC.

- Control+22 miembros, repartidos como sigue.

- 00/12 UTC: miembros 1-11 con horizonte de predicción 72 h, 12-22 con ciclo corto.

- 06/18 UTC: miembros 12-22 con horizonte de predicción hasta 72 h, 1-11 con ciclo corto.

- Reinicio cada 6 horas.

Es decir, sólo el control y la mitad de los 22 miembros funcionan con el horizonte de predicción completo, dejando miembros con ciclo corto de 7 horas para que se pueda mantener la continuidad. En el siguiente ciclo, los miembros que corrieron con el ciclo corto tendrán horizonte de predicción completo y viceversa. Así, el producto de los 22 miembros se podrá generar superponiendo los dos últimos ciclos completos.

La versión de WW3 que utilizará MetOffice incluye modificaciones para el manejo de las coordenadas polares giradas, una partición de olas revisada [4], advección de segundo orden [7] y una malla desestructurada (ver caja) SMC. 
Una malla desestructurada es un conjunto de polígonos que cubren una superficie con un patrón irregular. Habitualmente este tipo de mallas se utilizan en el método de elementos finitos (sec. 10.4 en la página 135) en regiones con límites irregulares, como la costa. Una malla Spherical Multi Cell o SMC [6] es una malla desestructurada con la particularidad de que conserva la rejilla en coordenadas cartesianas (latitud y longitud). Esta malla relaja la restricción CourantFriedrichs-Lewy (CFL) (cap. 10 en la página 129) en latitudes altas, uniendo varias celdas, y permite quitar puntos de tierra del modelo de oleaje, lo que reduce la advección en un $45 \%$. Todas las configuraciones usarán una resolución espectral con 24 direcciones y 30 frecuencias.

\subsubsection{Descripción del modelo local del Mediterráneo}

El sistema de Barcelona se fuerza con los campos de viento del Centro Nazionale di Meteorologia e Climatologia, CNMCA [1] y las condiciones de contorno del modelo Nettuno-EPS [1], descrito en el apartado 25.2.4. La malla donde están definidos estos forzamientos tendrá una resolución de $0.05^{\circ}(\mathrm{O} 2,05$, E 2,40, S 41,20, N 41,45).

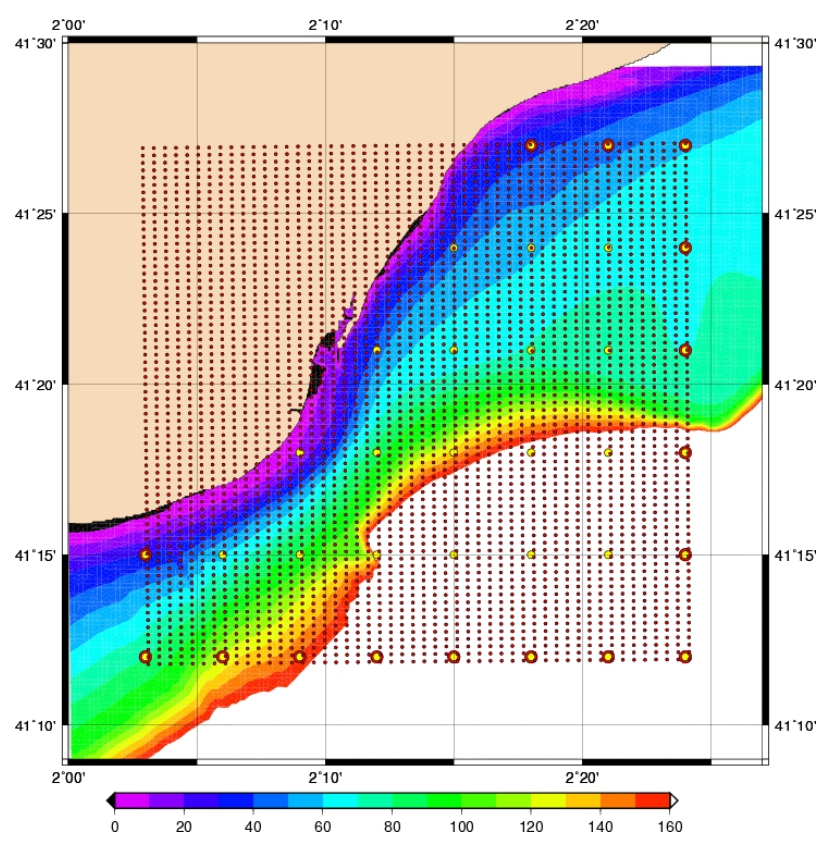

Figura 25.4: Configuración del sistema local WAVEEPS de Barcelona: malla computacional (puntos negros), campos de viento (puntos amarillos) y condiciones de contorno (puntos rojos). La batimetría (sombreado en colores, de 0 a 160 metros de profundidad), muestra los límites de aguas someras, y hasta dónde influiría en oleaje.
El sistema local se define en una malla computacional de 50x71 puntos y una resolución de 600x400 metros cada celda (Figura 25.4). Los campos de viento y condiciones de contorno tendrán una resolución de 3'.

\subsubsection{Descripción del wave-EPS del Me- diterráneo}

El Nettuno-EPS consiste en 40+1 miembros, integrados a las 00 UTC, con un horizonte de predicción de 48 horas en toda la cuenca mediterránea. El sistema corre en un ciclo diario a las 00 UTC. El modelo de oleaje que utiliza es el WAM [5], modelo operativo del ECMWF, y casi todas las instituciones europeas.

El sistema de Barcelona funcionará con la misma resolución temporal que el Nettuno-EPS. El modelo correrá de forma operativa con un ciclo al día, que empezará a las 08:30 UTC.

\subsection{Validación del sistema deter- minista de oleaje}

A partir de agosto de 2013, los 3 sistemas deterministas de oleaje para Barcelona, Gijón y Tenerife empezaron a funcionar de forma operativa.

El modelo operativo genera en cada ciclo series temporales en un punto específico con la velocidad, dirección de viento y los parámetros integrados Altura significativa de la ola, dirección media, periodo medio y periodo de pico. El punto elegido para estas series temporales será la localización de las boyas de Puertos del Estado, para poder comparar modelo con observación.

Parámetros integrados. Los parámetros integrados se calculan a partir de los espectros de densidad de varianza $E(f, \Theta)$.

$E$ (energía) $=$ aceleración_de_la_gravedad $\mathrm{x}$ densidad_del_agua $x$ espectro_densidad_de_varianza

- Altura significativa de la ola, $H_{s}=$ $4 \sqrt{\iint E(f, \Theta) d f d \Theta}$

- Dirección media, $\operatorname{Dir}=\arctan \left(\frac{\iint \sin \Theta E(f, \Theta) d f d \Theta}{\iint \cos \Theta E(f, \Theta) d f d \Theta}\right)$

- Periodo medio, $T m=\left(\frac{\iint f^{2} E(f, \boldsymbol{\Theta}) d f d \Theta}{\iint E(f, \boldsymbol{\Theta}) d f d \Theta}\right)^{-\frac{1}{2}}$ 


\begin{tabular}{|c|c|c|c|}
\hline Boya & Latitud & Longitud & Profundidad \\
\hline \hline Barcelona & $41,32^{\circ} \mathrm{N}$ & $2,20^{\circ} \mathrm{E}$ & $68 \mathrm{~m}$ \\
\hline S. C. Tenerife & $28,46^{\circ} \mathrm{N}$ & $16,23^{\circ} \mathrm{W}$ & $56 \mathrm{~m}$ \\
\hline
\end{tabular}

Tabla 25.1: Localización y profundidad de las boyas usadas para la validación.

En septiembre de 2013 la boya de Gijón dejó de funcionar, luego el estudio de validación que se muestra a continuación es el que se hizo sólo para las boyas de Tenerife y Barcelona, cuyos datos sobre emplazamiento y profundidad se presentan en la Tabla 25.1.

Las estadísticas presentadas a continuación (Figura 25.5 y Tabla 25.2 para Barcelona y Figura 25.6 en la página siguiente y Tabla 25.3 en la página siguiente para Tenerife) están basadas en los parámetros
Altura significativa y dirección media. Los gráficos de dispersión muestran el comportamiento del modelo a las $\mathrm{H}+0 \mathrm{~h}$ y $\mathrm{H}+48 \mathrm{~h}$ para Barcelona y a las $\mathrm{H}+0 \mathrm{~h}$, $\mathrm{H}+24 \mathrm{~h}, \mathrm{H}+48 \mathrm{~h}$ y $\mathrm{H}+72 \mathrm{~h}$ para Tenerife. Estos resultados dan una idea de cómo funciona el miembro 0 (modelo determinista), antes de desarrollar el sistema de predicción por conjuntos. Sobre el fundamento de las medidas estadísticas, puede consultarse la sección 15.2.3 en la página 211.
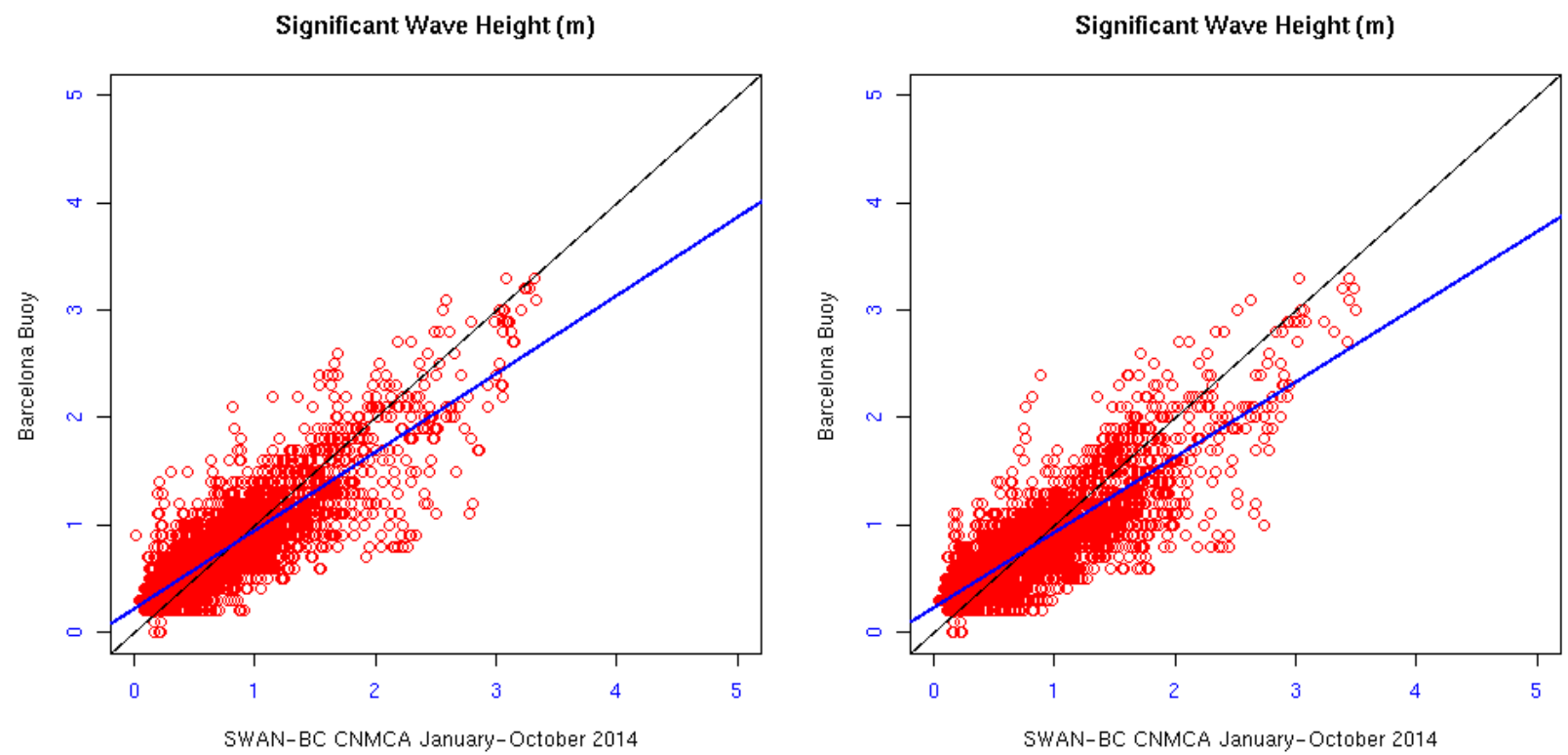

Figura 25.5: Barcelona-CNMCA: gráficos de dispersión de Altura significativa del oleaje para $T+0$ y $T+48$ h. Modelo determinista SWAN/boya de Barcelona.

\begin{tabular}{|c|c|c|c|c|c|c|c|}
\hline & Modelo & Num datos & Corr & Pendiente & RECM & EM & Dispersión \\
\hline \hline \multirow{3}{*}{ Altura significativa de ola } & SWAN - Barcelona & 4831 & 0,86 & 0,73 & 0,26 & 0,03 & 0,37 \\
\cline { 2 - 8 } & SWAN 48 -Barcelona & 4808 & 0,84 & 0,70 & 0,28 & 0,01 & 0,39 \\
\hline \hline \multirow{3}{*}{ Dirección media } & SWAN - Barcelona & 591 & 0,61 & 0,55 & 128 & $-34,5$ & 0,61 \\
\cline { 2 - 8 } & SWAN 24 -Barcelona & 583 & 0,63 & 0,57 & 126 & $-45,2$ & 0,59 \\
\cline { 2 - 8 } & SWAN 48 -Barcelona & 577 & 0,63 & 0,57 & 127 & $-45,2$ & 0,59 \\
\hline
\end{tabular}

Tabla 25.2: Barcelona-CNMCA: estadísticos de Altura significativa y dirección media del oleaje (Corr: coeficiente de correlación, RECM: raíz del error cuadrático medio, EM: error medio). Modelo determinista SWAN/boya de Barcelona. 

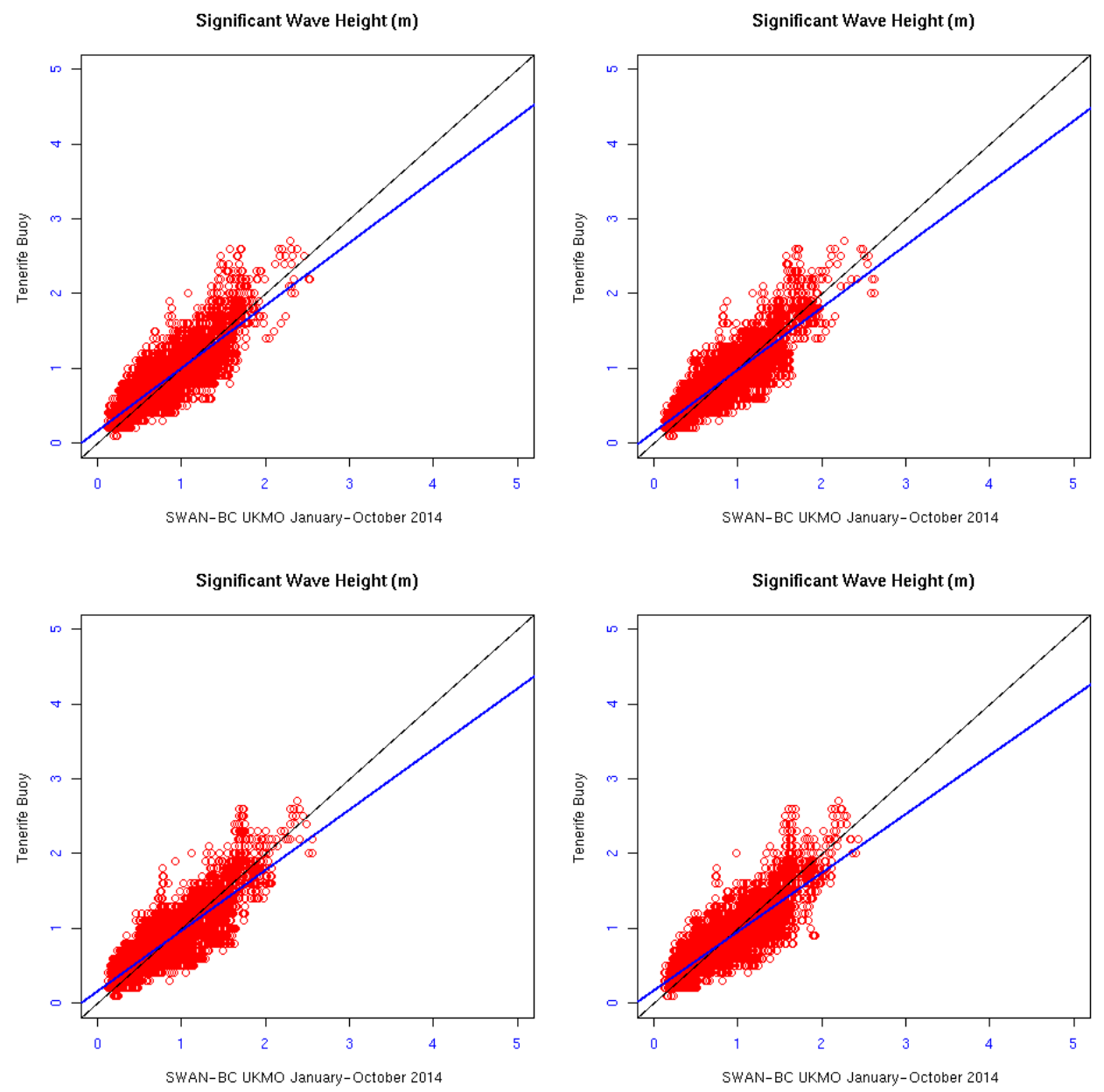

Figura 25.6: Tenerife-UKMO: gráficos de dispersión de Altura significativa del oleaje para T+0, 24, 48 y $72 \mathrm{~h}$. Modelo determinista SWAN/boya de Tenerife.

\begin{tabular}{|c|c|c|c|c|c|c|c|}
\hline & Modelo & Num datos & Corr & Pendiente & RECM & EM & Dispersión \\
\hline \hline \multirow{4}{*}{ Altura significativa } & SWAN - Tenerife & 6822 & 0,85 & 0,83 & 0,22 & 0,05 & 0,29 \\
\cline { 2 - 8 } & SWAN 24 - Tenerife & 6803 & 0,86 & 0,83 & 0,21 & 0,02 & 0,27 \\
\cline { 2 - 8 } & SWAN 48 - Tenerife & 6783 & 0,86 & 0,81 & 0,22 & 0,01 & 0,27 \\
\cline { 2 - 8 } & SWAN 72 - Tenerife & 6753 & 0,84 & 0,79 & 0,22 & 0,01 & 0,28 \\
\hline \hline \multirow{5}{*}{ Dirección media } & SWAN - Tenerife & 2665 & 0,69 & 0,62 & 32,6 & -19.0 & 0,12 \\
\cline { 2 - 8 } & SWAN 24 - Tenerife & 2646 & 0,69 & 0,64 & 32,1 & -17.6 & 0,12 \\
\cline { 2 - 8 } & SWAN 48 - Tenerife & 2642 & 0,75 & 0,72 & 30,3 & -17.4 & 0,12 \\
\cline { 2 - 8 } & SWAN 72 - Tenerife & 2632 & 0,72 & 0,72 & 30,0 & -16.3 & 0,11 \\
\hline
\end{tabular}

Tabla 25.3: Tenerife-UKMO: estadísticos como en la Figura 25.5 en la página anterior. Modelo determinista SWAN/boya de Tenerife.

\subsection{El modelo local WAVE-EPS para los puertos}

Una vez implementados los sistemas deterministas, se desarrollaron los sistemas por conjuntos para Gijón,
Tenerife y Barcelona. Los 3 sistemas locales WAVEEPS empezaron a funcionar de forma operativa en septiembre del 2013. Las Figuras 25.7 en la página siguiente y 25.8 en la página 380 muestran mapas de dispersión-promedio (sec. 27.5.1 en la página 412) en ambos sistemas. 


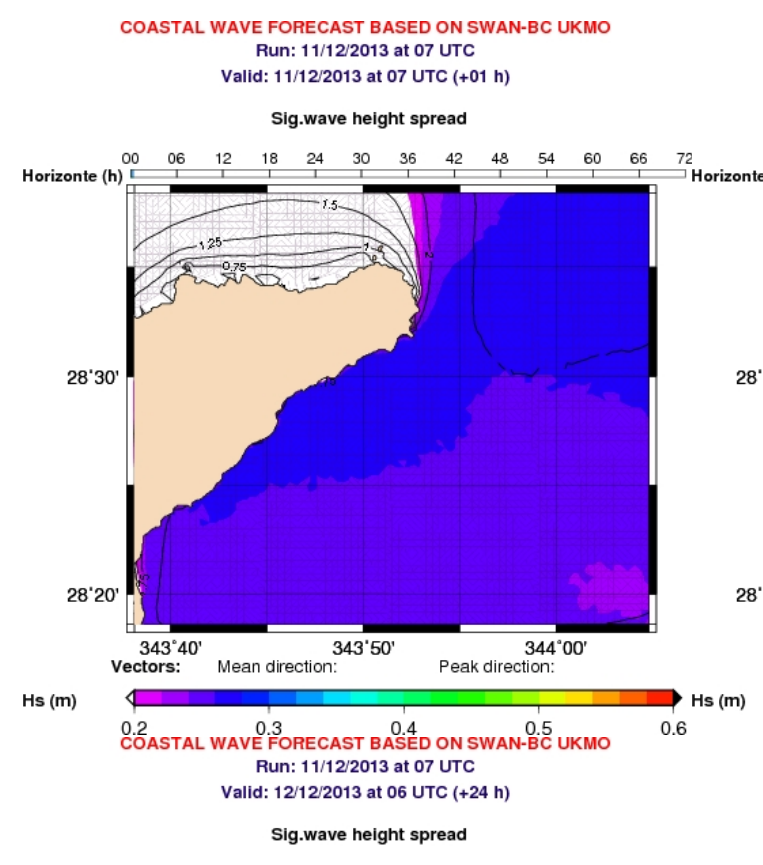
COASTAL WAVE FORECAST BASED ON SWAN-BC UKMO
Run: 11/12/2013 at 07 UTC Valid: 11/12/2013 at 18 UTC $(+12 \mathrm{~h})$

Sig.wave height spread

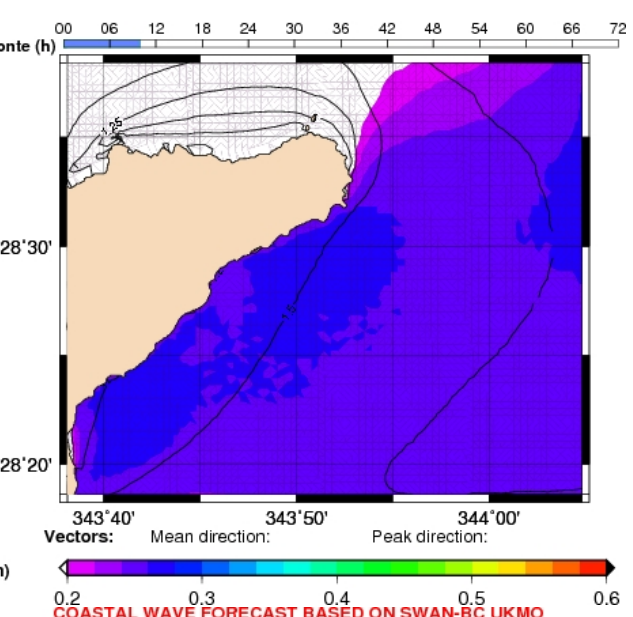

CO.2 ASTAL WAVE FORECAST BASED ON SWAN-BC UKMO Run: 11/12/2013 at 07 UTC Valid: 12/12/2013 at 18 UTC $(+36 \mathrm{~h})$

Sig.wave height spread
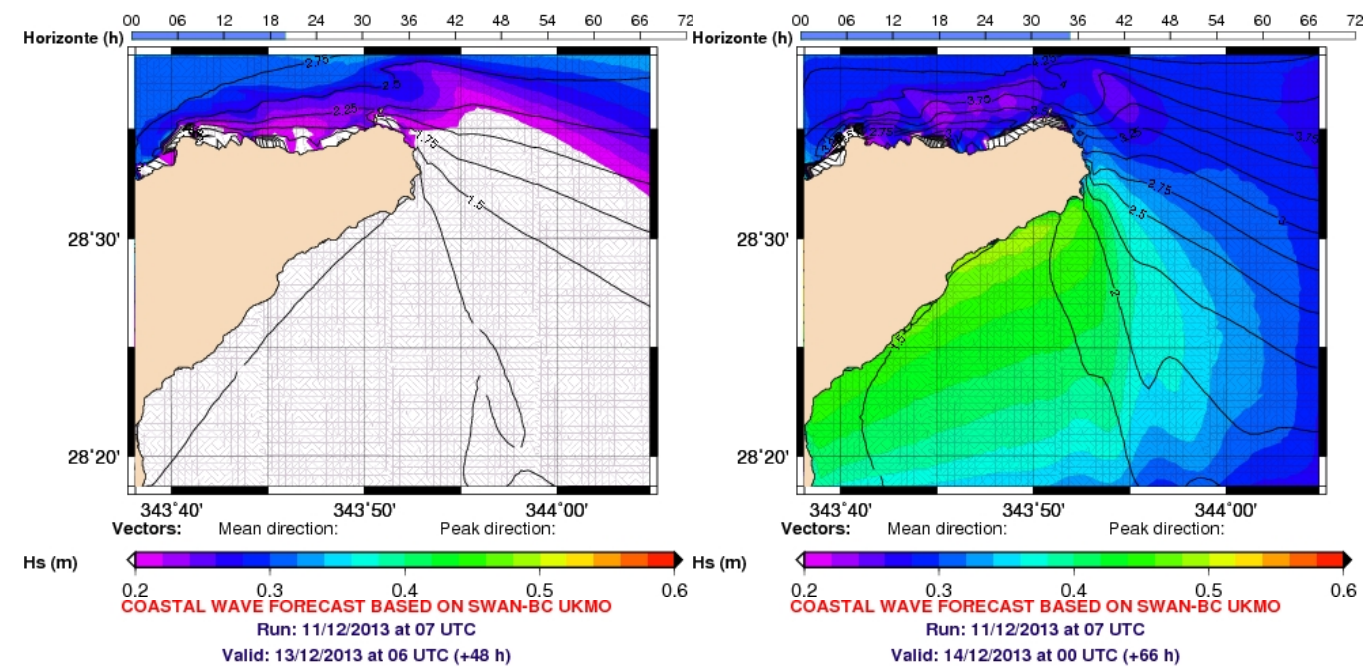
Sig.wave height spread
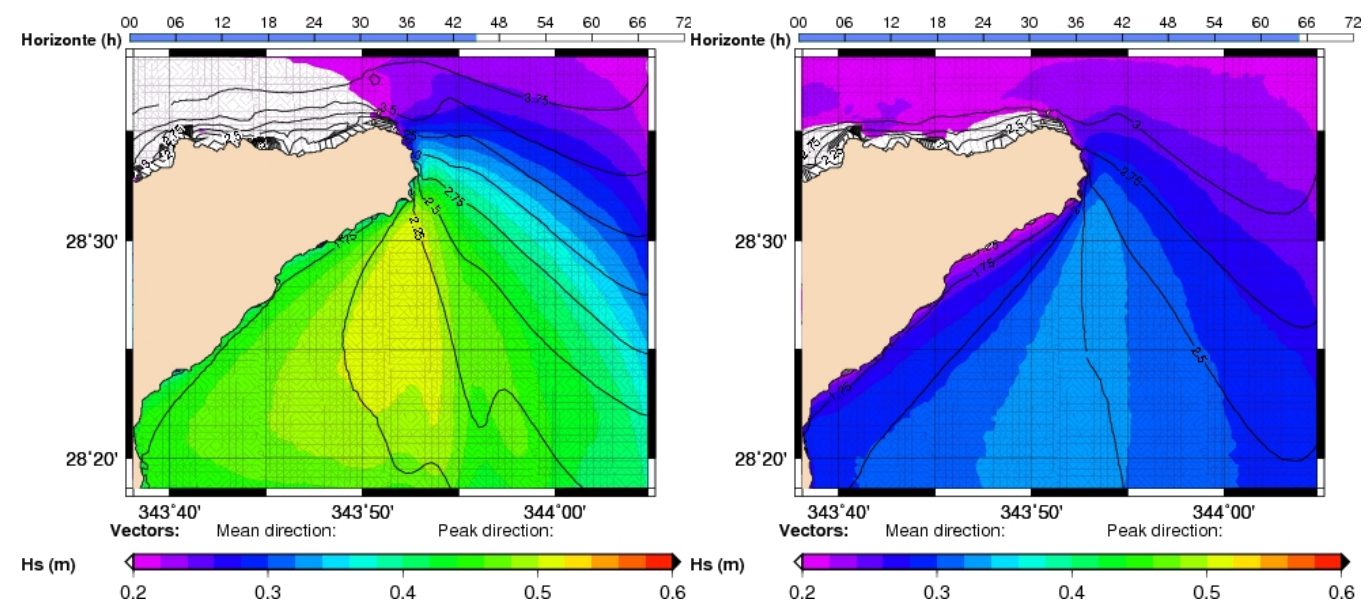

Figura 25.7: Mapas de dispersión-promedio del sistema de Tenerife, con el parámetro de Altura significativa de la ola, generados en la operativa cada ciclo de predicción. Las líneas de contorno indican la media del ensemble. 
COASTAL WAVE FORECAST BASED ON SWAN-BC UKMO
Run: $12 / 12 / 2013$ at 01 UTC Valid: 12/12/2013 at 01 UTC $(+01 \mathrm{~h})$

Sig.wave height spread
COASTAL WAVE FORECAST BASED ON SWAN-BC UKMO 2/12/2013 at 01 UTC

(2/12/2013 at 12 UTC $(+12$

Horizonte (h) $\begin{array}{lllllllllllllllllllllllllllll}00 & 06 & 12 & 18 & 24 & 30 & 36 & 42 & 48 & 54 & 60 & 66 & 72 \\ 1\end{array}$
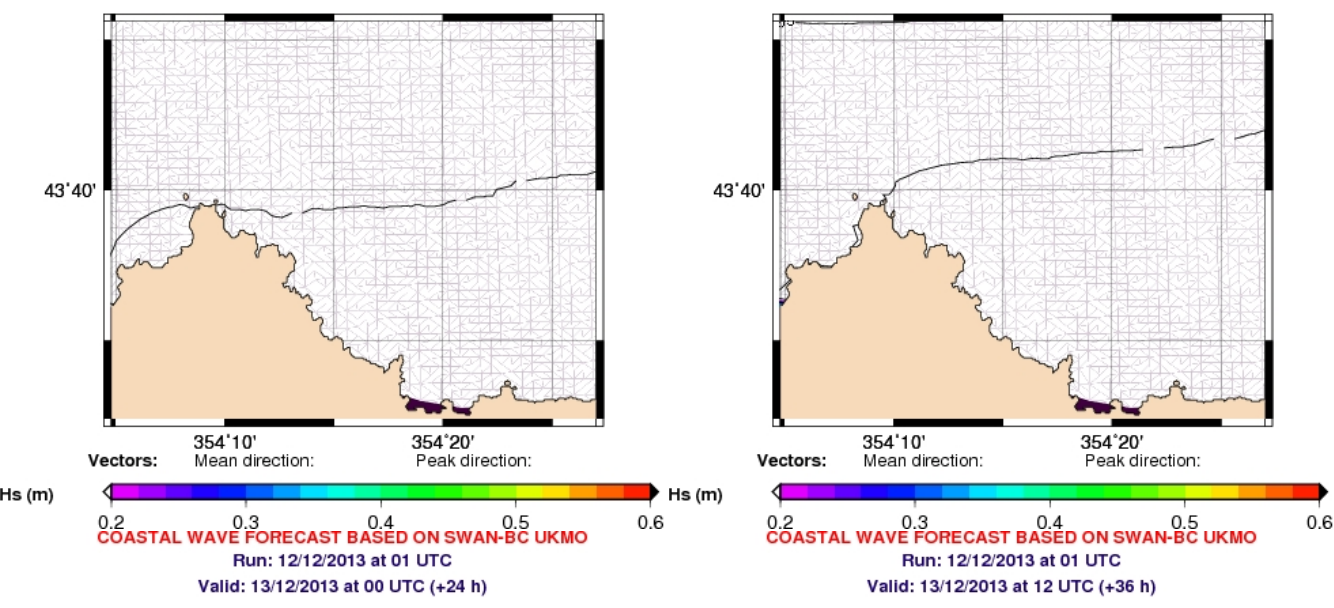

CÖASTAL WAVE FORECAST BASED ON SWAN-BC UKMO Run: $12 / 12 / 2013$ at 01 UTC Valid: $13 / 12 / 2013$ at 12 UTC $(+36 \mathrm{~h})$

Sig.wave height spread

Horizonte (h) \begin{tabular}{lllllllllllllllllllllllllll}
00 & 06 & 12 & 18 & 24 & 30 & 36 & 42 & 48 & 54 & 60 & 66 & 72 & 00 & 06 & 12 & 18 & 24 & 30 & 36 & 42 & 48 & 54 & 60 & 66 & 72 \\
\hline
\end{tabular}
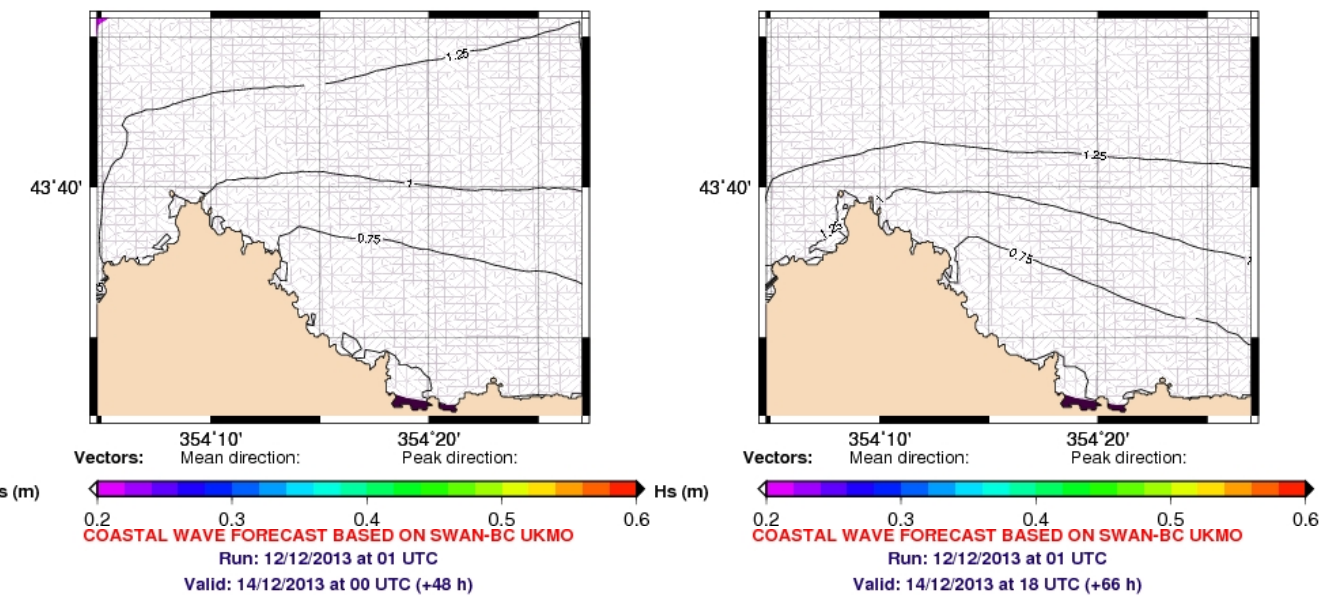

Sig.wave height spread

Sig.wave height spread

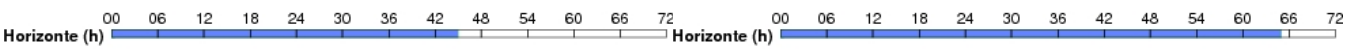
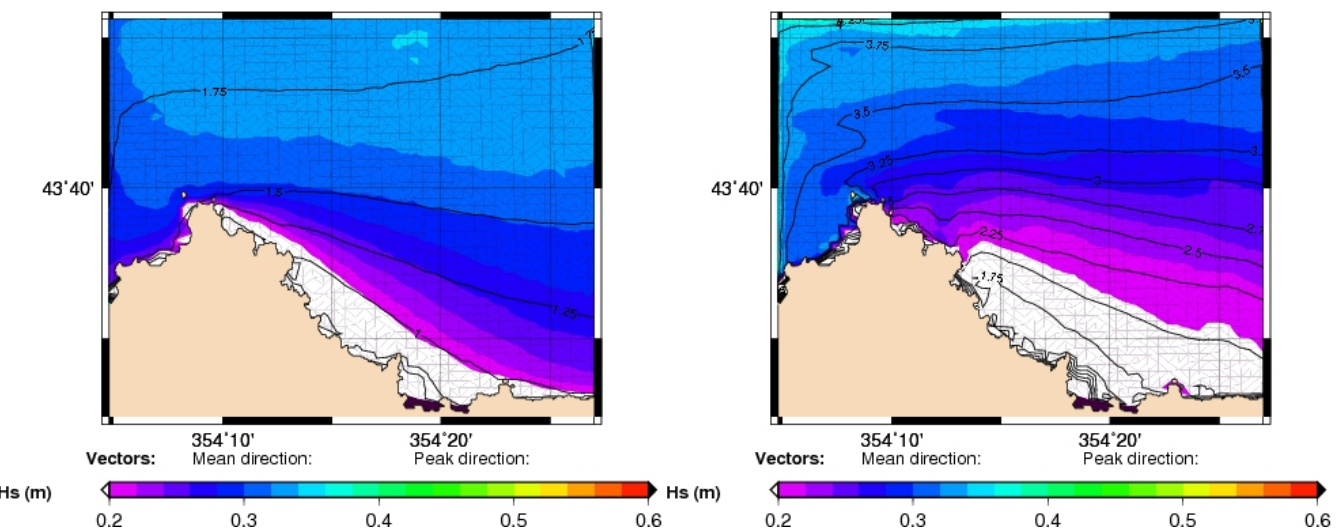

Figura 25.8: Mapas de dispersión-promedio del sistema de Gijón, con el parámetro de Altura significativa de la ola, generados en la operativa cada ciclo de predicción. Las líneas de contorno indican la media del ensemble. 


\subsubsection{Consistencia del WAVE-EPS}

Para estimar la fiabilidad con una significación del $10 \%$ de un SPC de 20 miembros, se necesitarían aproximadamente 14000 salidas del modelo. Partiendo de esta base, es recomendable poder hacer la verificación de un periodo de al menos 3-6 meses. En este caso, se tomaron los resultados de la salida del modelo de enero a mayo de 2014 (4 meses).

El primer paso en la validación del WAVE-EPS, como cualquier sistema de predicción probabilista, es comprobar su consistencia estadística con las observaciones (sec. 15.8 en la página 222). El histograma de rango (sec. 15.8.1 en la página 222) se usó para comprobar si la observación es estadísticamente indistinguible del conjunto de valores pronosticados.

Se puede observar cómo el sistema de Barcelona WAVE-EPS (Figura 25.9) comienza con una dispersión baja que va aumentando a medida que aumenta el horizonte de predicción, con un histograma de bajo rango dispersivo para $\mathrm{H}+06$ y consistente para $\mathrm{H}+24$ y $\mathrm{H}+48$.

El histograma de Tenerife WAVE-EPS (figura 25.10), muestra un sistema estadísticamente consistente, con tendencia a la sobrepredicción (bias positivo).

Para completar la información sobre la validación de los WAVE-EPS locales en este apartado, se muestra a continuación un resumen con las series temporales o espagueti.

Estos diagramas presentan la evolución de la predicción por conjuntos del parámetro de Altura significativa con una mayor dispersión conforme va aumentando el horizonte, algo que se muestra de forma clara en la primera gráfica (Figura 25.11 en la página siguiente, modelo Barcelona WAVE-EPS). En la segunda se presenta Gijón (Figura 25.12 en la página siguiente, izquierda). La última gráfica (Figura 25.12 en la página siguiente, derecha), espagueti del modelo de Tenerife, muestra cómo la observación (en verde) está dentro de la dispersión del modelo.
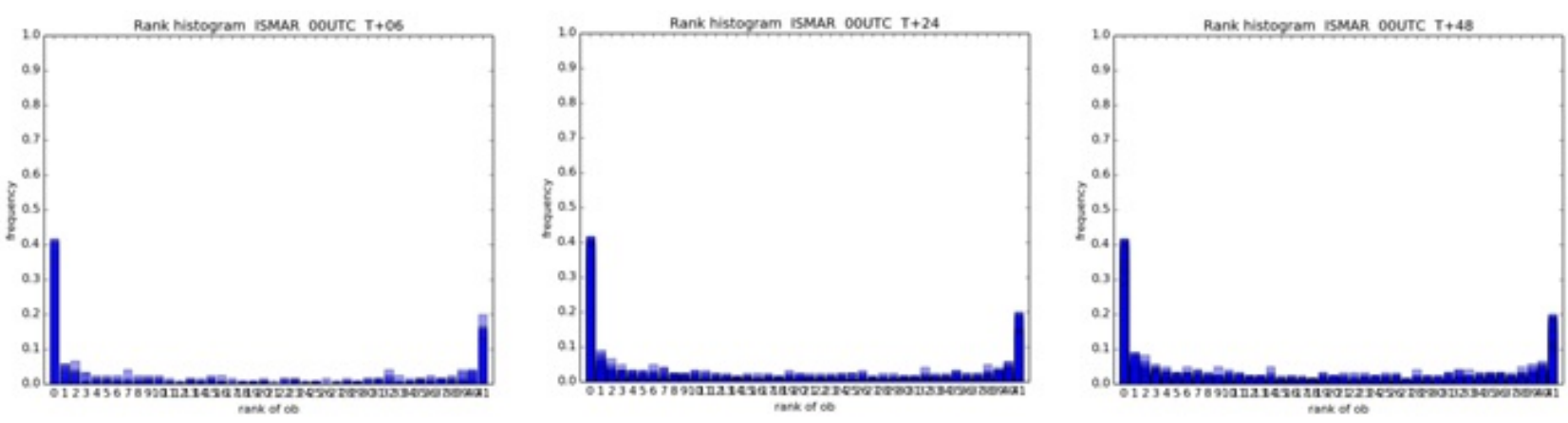

Figura 25.9: Histogramas de rango con la Altura significativa del sistema Barcelona WAVE-EPS, con horizonte de predicción $T+06, T+24, y T+48$.
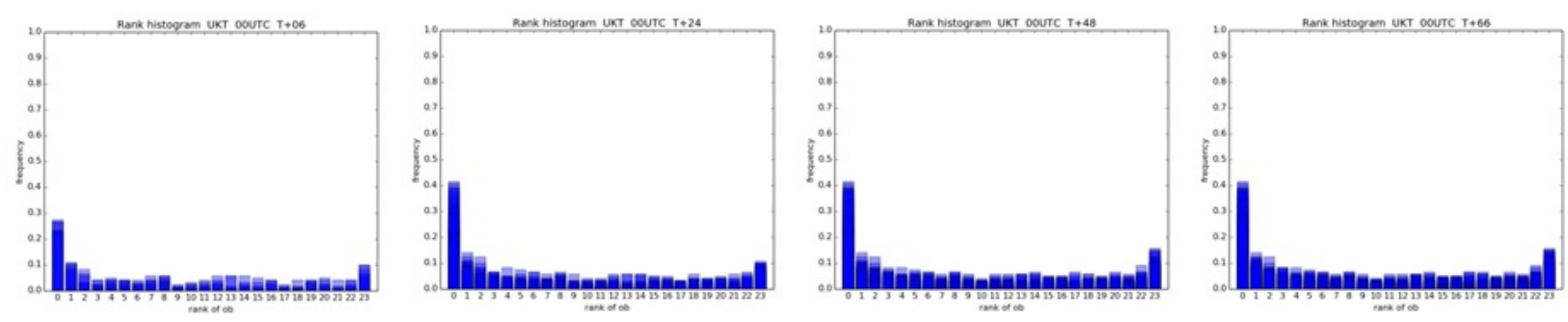

Figura 25.10: Histogramas de rango con la Altura significativa del sistema Tenerife WAVE-EPS, con horizonte de predicción T+06, T+24, T+48 y T+66. Para el cálculo del histograma, también se usaron los datos del ciclo corto. 

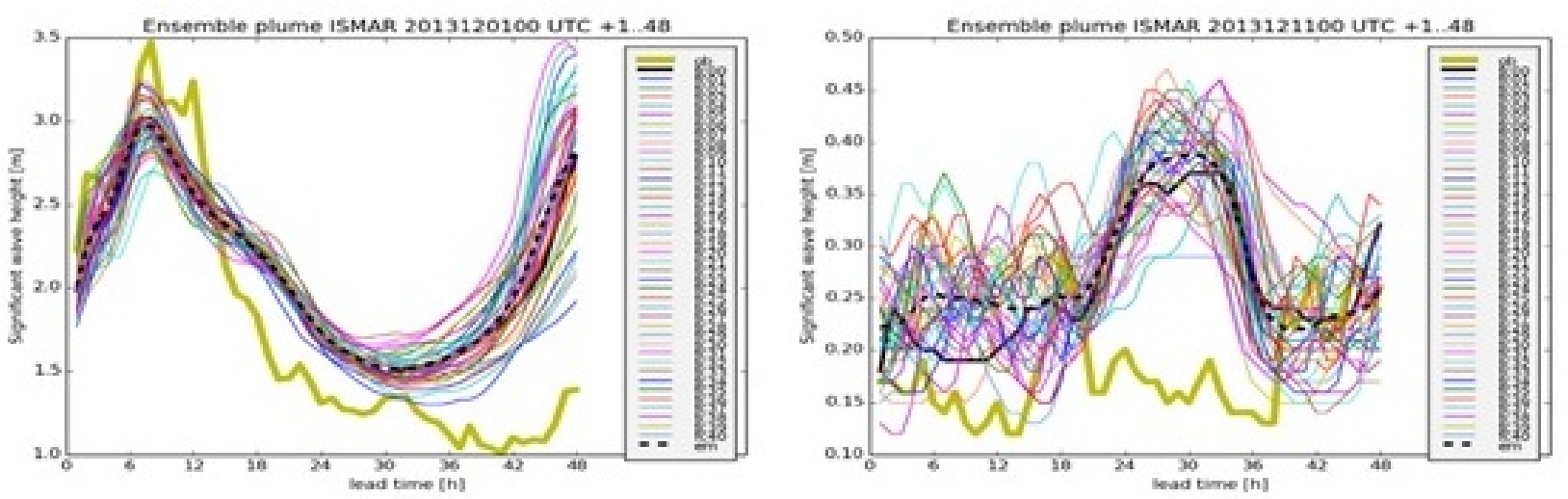

Figura 25.11: Series temporales (espagueti) de los WAVE-EPS de Barcelona (arriba). En verde, la observación, y en negro, el miembro de control.
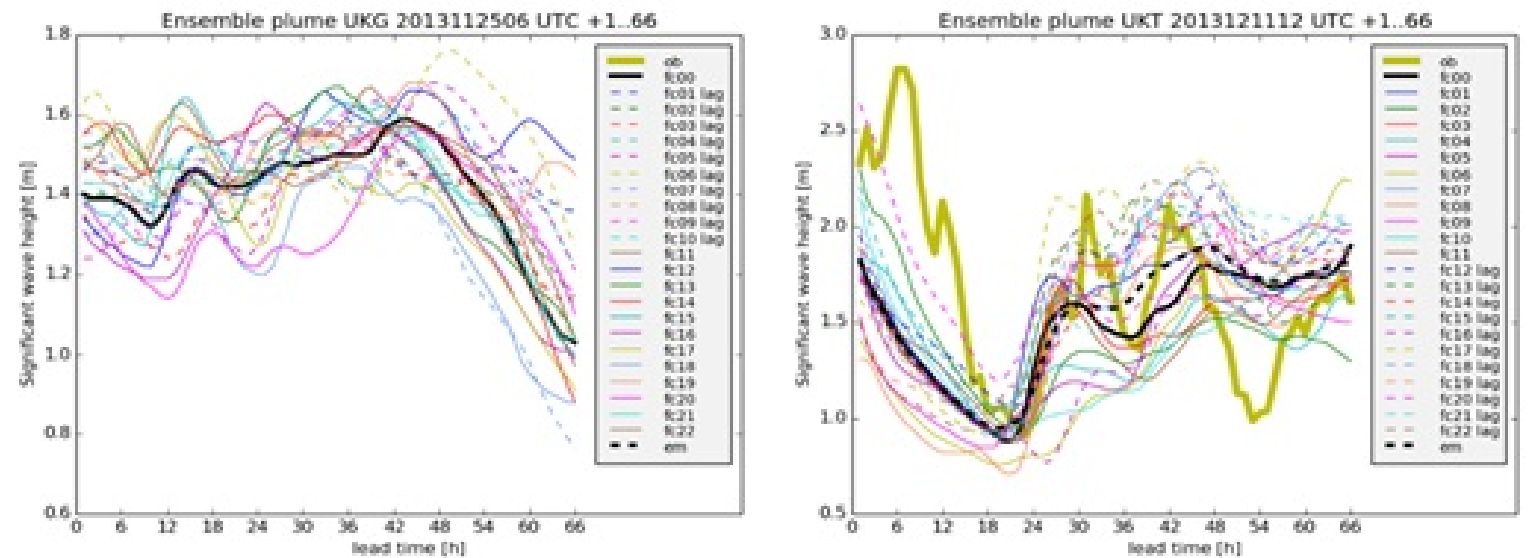

Figura 25.12: Series temporales (espagueti), como en la Figura 25.11, de los WAVE-EPS de Gijón (izquierda) y Tenerife (derecha).

\subsection{Resultados}

Los dos productos principales de la predicción de oleaje por conjuntos para Gijón, Barcelona y Tenerife fueron EPSgramas (sec. 27.8.1 en la página 425) y mapas de probabilidad (sec. 27.6.1 en la página 416). En este apartado se muestran ejemplos para los tres puertos a continuación.

Un EPSgrama muestra la evolución temporal de la distribución estadística de los miembros del ensemble en una localización geográfica determinada (sec. 27.8.1 en la página 425). Los EPSgramas de oleaje (Figuras 25.13 en la página siguiente y 25.14 en la página 384) nos dan la distribución de los parámetros Altura significativa y Dirección Media en un punto concreto.

La dispersión está calculada por el WAV-EPS. El 50\% de los miembros se distribuyen uniformemente alrede- dor de la mediana para definir un rectángulo vertical. Los miembros restantes definen los picos extremos del $25 \%$. El EPSgrama de cajas proporciona así una información probabilista discreta en los intervalos $0-25 \%$, $25-50 \%$ y $75-100 \%$, con una resolución temporal de 3 horas. El miembro determinista (miembro de control) se incluye como referencia. El gráfico con el EPSgrama continuo tiene resolución horaria, que es la resolución de la salida de los 3 sistemas locales implementados.

Como el nombre indica, los mapas de probabilidad (sec. 27.6.1 en la página 416) muestran la probabilidad de que un determinado parámetro exceda un umbral dado. La Figura 25.15 en la página 384 muestra las probabilidades de que la Altura significativa de la ola supere los 4,5 metros en el puerto de Gijón y la Figura 25.16 en la página 385 los 1,8 metros en Barcelona. 
Forecast base time: 20141114 oo UTC Members:22

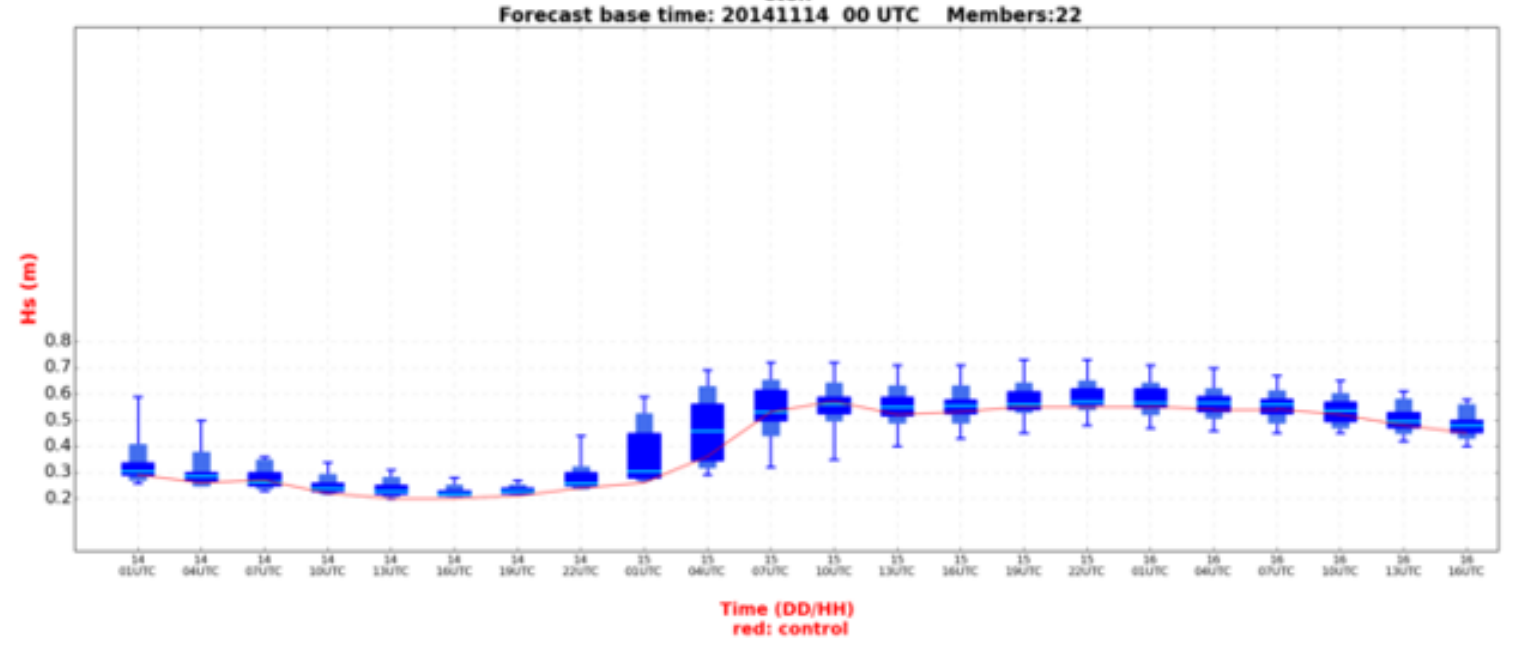

Cten
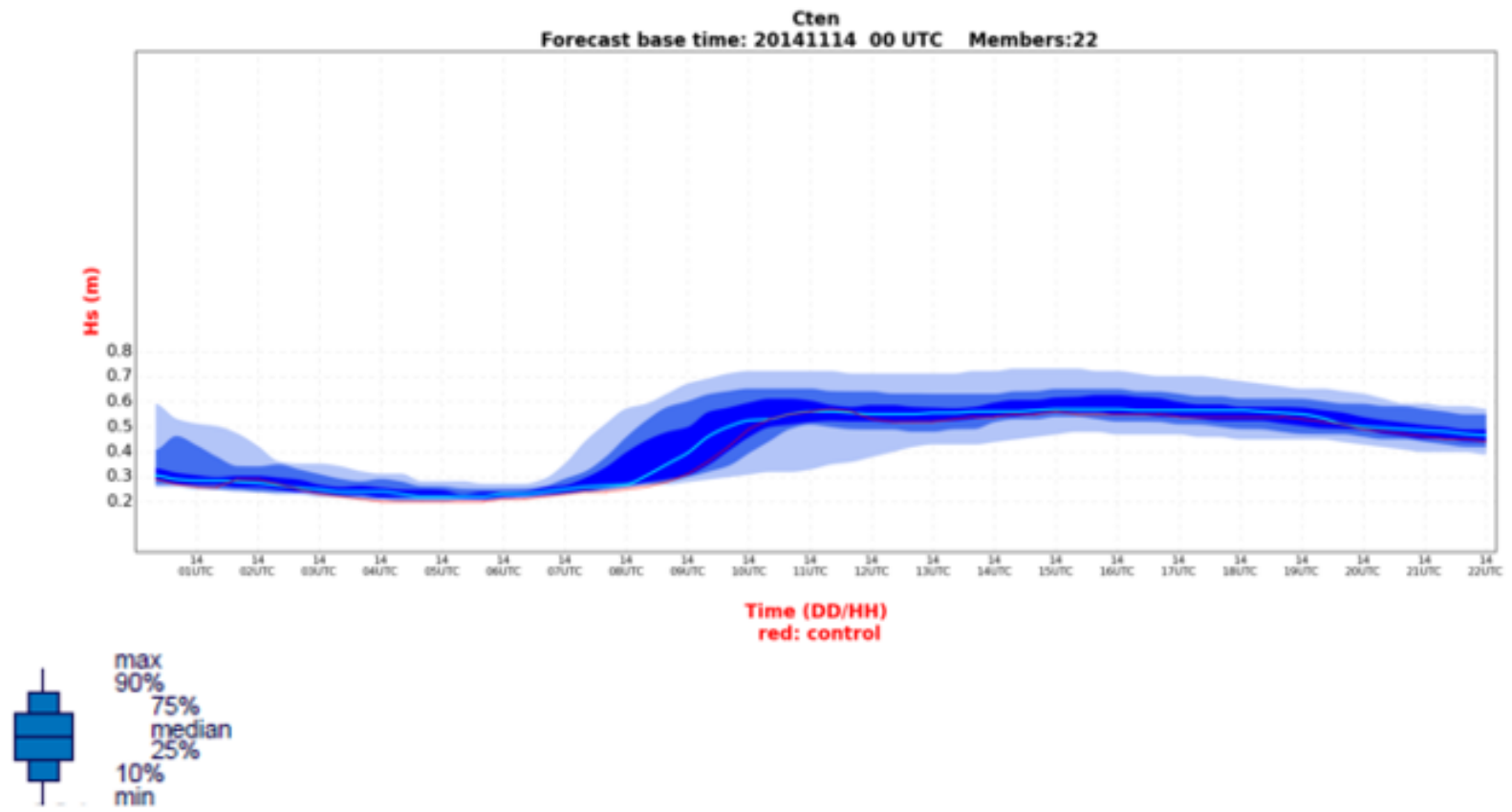

Wave direction Cten Forecast base time: 20141114 00 UTC Members:22
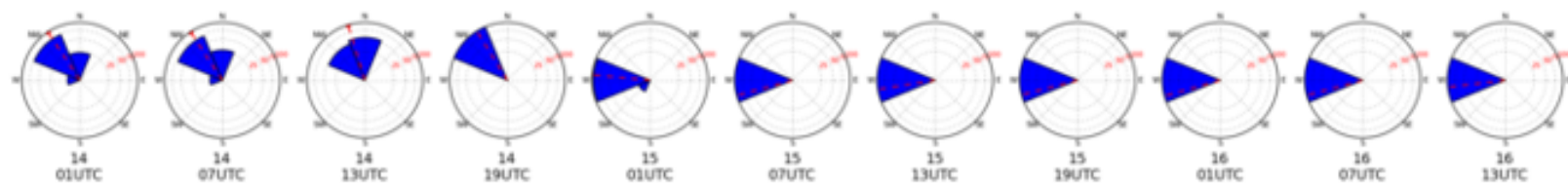

Figura 25.13: Ejemplo de EPSgramas para Tenerife, con la Altura significativa y dirección media, ciclo 2014111400. 

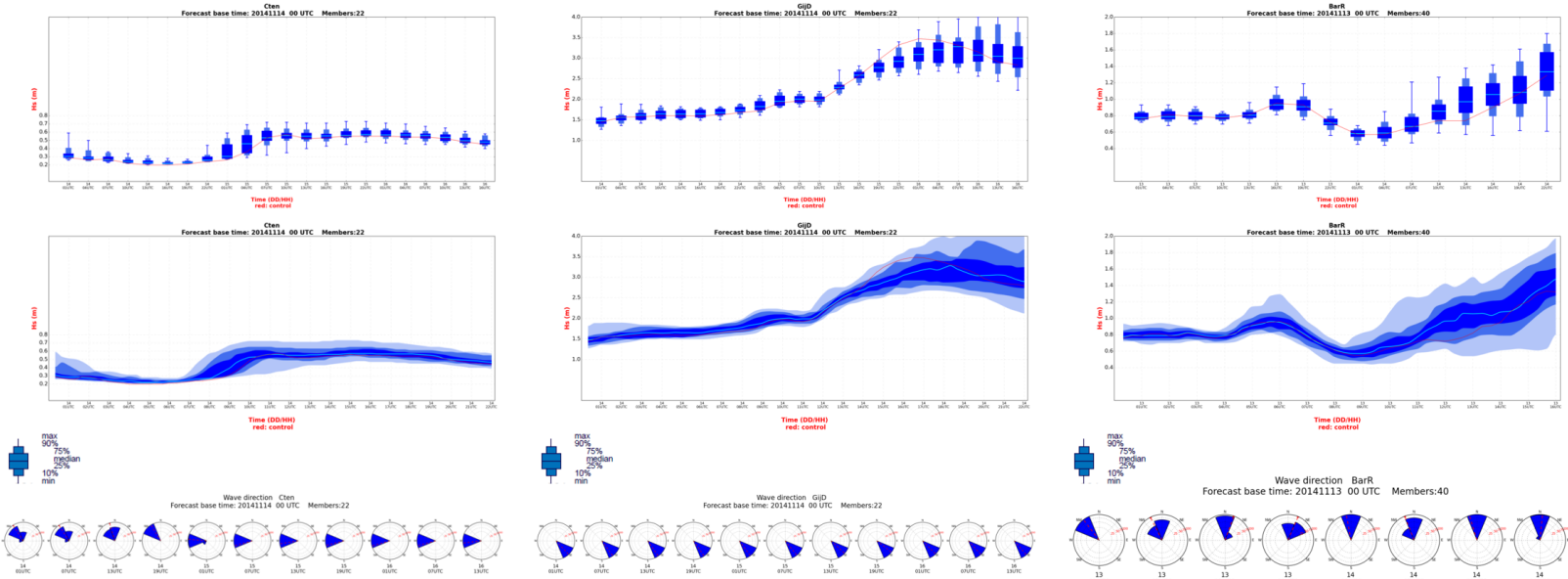

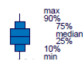

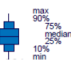
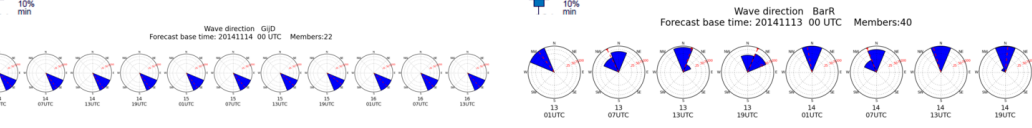

Figura 25.14: Ejemplo de EPSgramas para Tenerife (izquierda, mostrado anteriormente en la Figura 25.13 en la página anterior) y, para comparar en términos generales y sin entrar en detalle, Gijón (centro) y Barcelona (derecha), todos ellos con la Altura significativa y dirección media, ciclo 2014111400.

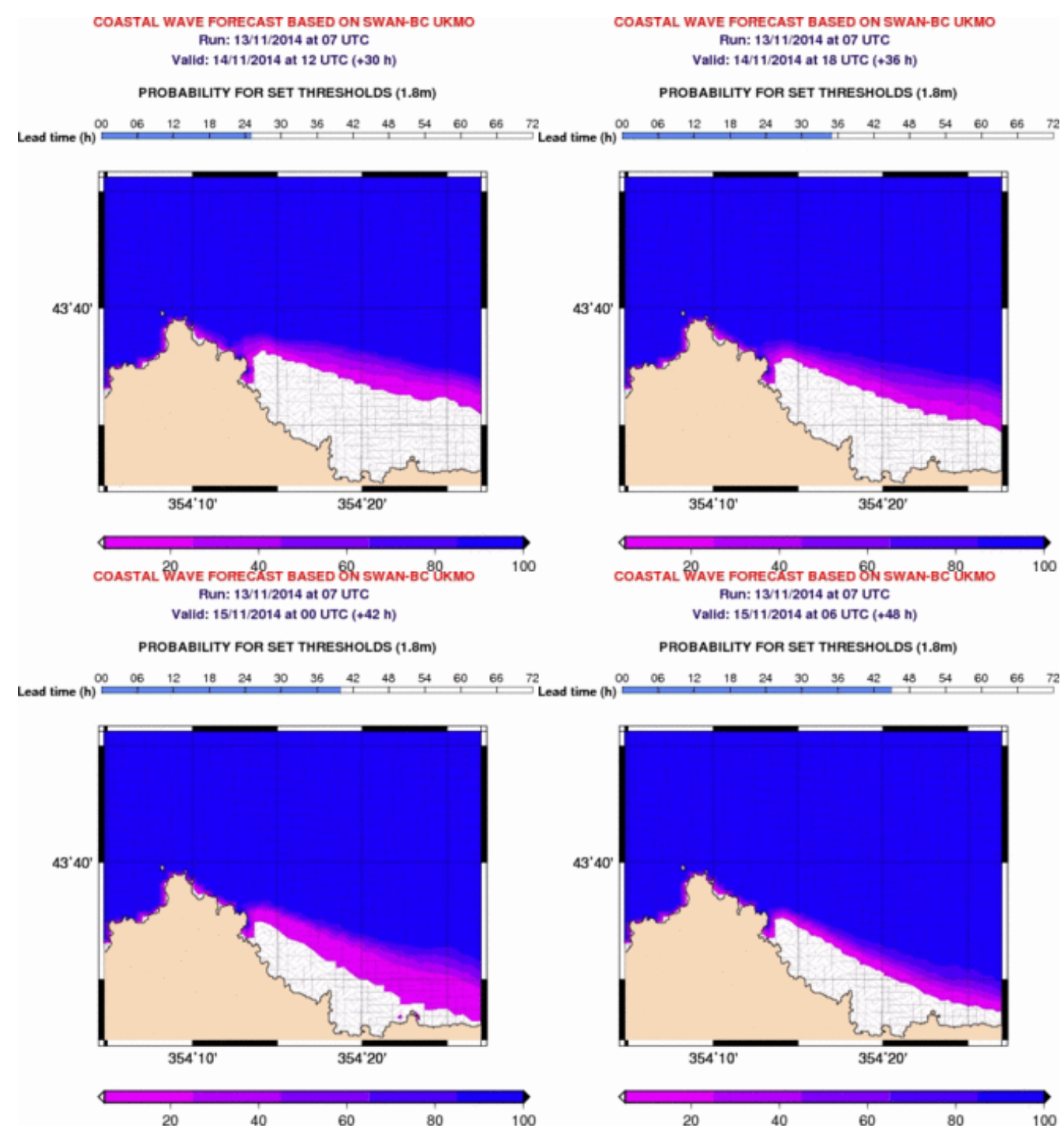

Figura 25.15: Mapas de probabilidad para Gijón, umbral de Altura significativa: 4,5 metros. 


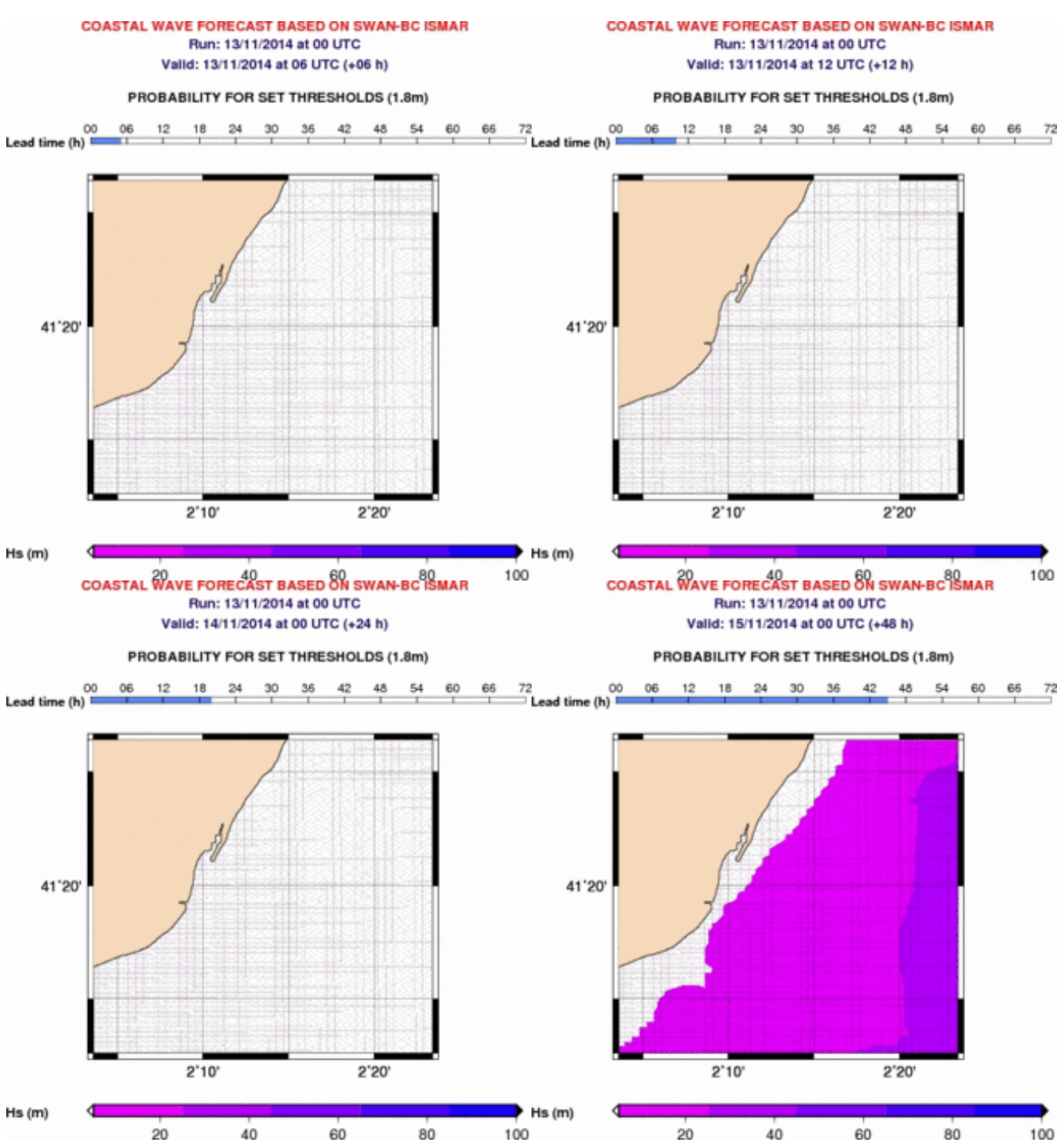

Figura 25.16: Mapas de probabilidad para Barcelona, umbral de Altura significativa: 1,8 metros.

\subsection{Conclusiones}

Durante los años de trabajo del proyecto, 2012-2014, apenas había comenzado a aplicarse la predecibilidad en los modelos de oleaje. La generación de sistemas de predicción por conjuntos proporcionó nuevos productos y nuevas opciones de visualización de la información, enriqueciendo así las técnicas de predicción del oleaje.

Los sistemas locales de WAVE-EPS se pusieron operativos en septiembre de 2013 con el objetivo principal de proporcionar predicciones categóricas con la mayor exactitud posible y una base cuantitativa para un pronóstico de probabilidad fiable y útil.

La información probabilista fue adaptada a las necesi- dades de Barcelona, Gijón y Tenerife. Se incluyó en la operativa rutinas para generar EPSgramas, mapas de probabilidad y de dispersión-promedio.

Los mapas de probabilidad con el umbral específico de alerta para cada autoridad portuaria son una herramienta importante para agregar al sistema determinista que usan ahora los puertos.

Aunque los modelos locales de WAVE-EPS podrían generar más productos para los puertos comerciales, los EPSgramas con la dirección media o la Altura significativa y los mapas de probabilidad son las herramientas más útiles para comenzar a introducir información sobre predecibilidad en la meteorología marítima. 


\subsection{Referencias}

[1] Bertotti, Luciana y col. "Nettuno: Analysis of a Wind and Wave Forecast System for the Mediterranean Sea". En: Monthly Weather Review 141.9 (sep. de 2013), páginas 3130-3141. ISSN: 0027-0644. DOI: 10. 1175/MWR-D-12-00361.1 (citado en página 376).

[2] Booij, N., Holthuijsen, L.H. y Ris, R.C. "The Swan Wave Model for Shallow Water". En: Coastal Engineering 1996. New York, NY: American Society of Civil Engineers, ago. de 1997, páginas 668-676. ISBN: 9780784402429. DOI: 10 . $1061 /$ 9780784402429 . 053 (citado en página 374).

[3] Bowler, Neill E. y col. "The MOGREPS short-range ensemble prediction system". En: Quarterly Journal of the Royal Meteorological Society 134.632 (abr. de 2008), páginas 703-722. ISSN: 00359009. DOI: 10 . 1002/qj . 234 (citado en página 375).

[4] Bunney, Chris y Saulter, Andy. "An ensemble forecast system for prediction of Atlantic-UK wind waves". En: Ocean Modelling 96 (dic. de 2015), páginas 103-116. ISSN: 14635003 . DOI: $10.1016 / \mathrm{j}$. ocemod. 2015.07.005 (citado en página 375).

[5] Komen, G. J. Dynamics and modelling of ocean waves. Cambridge University Press,
1994. ISBN: 9780521577816 (citado en página 376).

[6] LI, Jian-Guo. "Propagation of ocean surface waves on a spherical multiple-cell grid". En: Journal of Computational Physics 231.24 (oct. de 2012), páginas 8262-8277. ISSN: 00219991. DOI: 10 . 1016/j . jcp . 2012 . 08.007 (citado en página 376).

[7] LI, Yineng y HuAng, Ping. “A coupled lattice Boltzmann model for advection and anisotropic dispersion problem in shallow water". En: Advances in Water Resources 31.12 (dic. de 2008), páginas 1719-1730. ISSN: 03091708. DOI: $10.1016 / \mathrm{j}$. advwatres . 2008.08.008 (citado en página 375).

[8] Pezzutto, Paolo y col. "Performance comparison of meso-scale ensemble wave forecasting systems for Mediterranean sea states". En: Ocean Modelling 104 (ago. de 2016), páginas 171-186. ISSN: 14635003. DOI: $10.1016 / \mathrm{j}$. ocemod. 2016.06.002 (citado en página 374 ).

[9] SAETRA, Øyvind. MyWave project final report. Informe técnico. FP7-SPACE, 2016. URL: http: //hdl . handle. net/20.500 . 11765/7249 (citado en página 374).

[10] Tolman, Hendrik L y col. "User manual and system documentation of WAVEWATCH III TM version 3.14". En: Technical note, MMAB Contribution 276 (2009), página 220 (citado en página 375 ). 
